\title{
Signal Transduction in Plants under Drought and Salt Stress-An Overview
}

\author{
Swapan K. Tripathy ${ }^{1 *}$, Gyanisha Nayak ${ }^{2}$, Jagruti Naik², Monalisa Patnaik ${ }^{2}$, \\ Asit P. Dash ${ }^{2}$, Dibya B. Sahoo ${ }^{2}$ and A.M. Prusti ${ }^{2}$ \\ ${ }^{1}$ Department of Agricultural Biotechnology, College of Agriculture, OUAT, \\ Bhubaneswar, India \\ ${ }^{2}$ Department of Plant Breeding and Genetics, College of Agriculture, OUAT, \\ Bhubaneswar, India \\ *Corresponding author
}

\section{A B S T R A C T}

\section{Keywords}

Drought and salt stress, Signal perception, Signal transduction pathways,

Relay proteins,

Abiotic tolerance

Article Info

Accepted:

04 July 2019

Available Online:

10 August 2019
Environmental factors that plants face during their life cycle are dynamic in nature. Plants require adequate amount of water for their growth and development. At the event of moisture deficit in the soil, the water potential in the cells drastically reduced. Under salt stress, plants succumb to salt injuries in addition to dehydration. Plants try to combat such situation by adopting a variety of mechanisms, including closure of stomata that limits water loss and exclusion of $\mathrm{Na}^{+}$to reduce salt injury. The process starts with stress signal perception, transduction of signal following different pathways and ultimately activation of regulatory proteins or even stress responsive genes to combat the hindrance to normal growth and development caused due to moisture deficit. In the present review, the authors presented a detailed overview of the signaling process in plants under drought and salt stress.

\section{Introduction}

Signal transduction systems are especially important in multicellular organisms for communication of their activities across hundreds of cells in response to various stimuli/stressors. In fact, plant needs such a fascinating signaling mechanism in every stage of its life cycle. It has role in morphogenetic development (overcoming dormancy, germination, growth, flowering, and fruiting), inter- cellular transport (nutrients, water, gaseous substances), cellular metabolism, response to nutritional $(\mathrm{Fe}, \mathrm{Zn}$, Bo, heavy metals), environmental (heat, cold, flooding) and biotic stresses (fungus, bacteria, virus causing diseases). Many signals interact co-operatively and synergistically with each other to produce the final response (Menon and Durakovic, 2014). Besides, it also decides senescence, apoptosis in plants and even symbiotic process of living together (e.g., 
endophytic bacteria on phytoplane and root zone) and deriving benefit from each other. The entire sequence comprising signal perception- intracellular signal transductioncellular response-is referred to as a signal transduction pathway.

\section{Signaling mechanisms in plants}

Sugars and hormones are the most common signalling molecules (ligand) in vascular tissue differentiation in plants. Besides, cytokinins, abscisins, salicylic acid, ethylene, and gibberellins, as well as brassinosteroids, jasmonates and plant hormones add to morphogenetic and biochemical complexity in plants. The major plant hormone induced and accumulated in plant tissues in response to moisture deficit is abscisic acid (ABA). High level of $\mathrm{ABA}$ in cell is related to drought tolerance. The signaling molecule (ligand) or even the environmental stresses are first perceived by sensor molecules (transmembrane receptor proteins) in the plasma membrane. Three major type of receptors (primary messengers) present in the plasma membrane are G-protein linked receptors, protein-kinase receptors (tyrosinekinases, histidine kinases or serine/threonine kinases) and ion channel receptors. In the later case, the ligands upon attachment with receptor proteins cause a conformational change (change in shape) of the receptor site forming pores (ion channels). These protein pores on the plasma membrane are opened once the ligand attaches the pore and thereby it allows free entry of $\mathrm{Ca}^{+2}$ into the cell in exchange of $\mathrm{Na}+$ ions that are excluded. The receptor proteins are activated by phosphorylation cascade. The molecules that relay a signal are often proteins. However, non-protein molecules like ions $\left(\mathrm{Ca}^{+2}\right.$ and $\left.\mathrm{H}^{+}\right)$ (Yang and Poovaiah, 2003), nucleotides (Basu et al., 2010) and phospholipids (Eyster, 2007) can also play as messengers. Phosphate groups can't be attached to just any part of a protein.
Instead, they are typically linked to one of the three amino acids that have hydroxyl $(-\mathrm{OH})$ groups in their side chains: tyrosine, threonine, and serine. The transfer of the phosphate group is catalyzed by enzymes called a protein kinases, and cells contain many different kinases that phosphorylate different targets. Protein kinases transfer phosphate group from ATP to another receptor protein. to trigger responses (almost any cellular activity) and these transformed molecules interact with the information-relaying small molecules e.g., $\mathrm{Ca}^{+2}$, Cyclic AMP (cAMP), Cyclic GMP(cGMP), diacylglycerol and inositol triphosphate (secondary messengers) in the cytoplasm to carry forward the signal in a series of steps to reach the target gene and causes its expression or repression. In general, phosphorylation isn't permanent. To flip proteins back into their non-phosphorylated state, cells have enzymes called phosphatases, which remove a phosphate group from ATP and convert it to ADP and inorganic phosphate pi as bi-products.

Besides, the cellular responses are manifested in terms of change in ion permeability, or a change in the enzyme activity or protein threedimensional structure, which ultimately affects the metabolism of the cell or organism. Signal dependent protein degradation is also a prevalent regulatory mechanism in plants. A single cell may have several types of receptors each binding to a specific signal molecule and a cell can receive a number of different types of signal molecules (ligands) simultaneously. Some of the plant hormones, lipid-soluble molecules such as steroids (steroid hormones) or small molecules (such as nitric oxide) can directly enter into the cytoplasm where they bind with free receptor protein in the cytoplasm. Such complex move directly (without involvement of secondary messengers) to the nucleus to activate transcriptional factors (TFs) and help transcription of certain target genes. 
In Escherichia coli, almost $90 \%$ of the genes $(27,000)$ are thought to provide protection against randomly varying environment condition through signal transduction. The situation becomes more complex in higher plants which face vagaries of environmental stresses(cold, high temperature, salinity, eat stress etc), pathogens, pests and herbivores throughout their life cycle to ensure better survivability. In Arabidopsis genome, as many as 1800 genes encoding transcription factors, more than 600 genes encoding protein kinases and 600 genes encoding F-box proteins are having role in signalling process. In addition, post transcriptional regulation at RNA level also leads to various other signalling pathways.

\section{Plant responses to drought stress}

Water deficit reduces crop yield regardless of the varieties owing to energy (ATP) drain to combat drought which could otherwise be utilized to increase sink size. Water deficit in root zone causes an increase in rate of root respiration which leads to an imbalance in the utilization of carbon resources, decrease in production of adenosine tri-phosphate (ATP) and an increase in production of $\mathrm{ABA}$ and reactive oxygen species (ROS). ABA is transported via the transpiration stream to the leaves through xylem. Concentration of ABA in the guard cell apoplast increased as a stress signal and it activates a set of receptor proteins leading to a set of changes and restricts stomatal opening and shoot growth. But, root growth appears to be promoted to absorb water from soil. A number of genes are elicited under abiotic stresses. Now a days, different versions of $\mathrm{ABA}$ receptor genes are being tried to tune closure of guard cells. Besides, status of drought tolerance depends upon changes of Rubisco and some stress inducible proteins (chaperons, HSPs, dehydrins and proteases) and their complex interrelationships under drought. Dehydrin protects cytosolic structures from the deleterious effects of cellular dehydration.

\section{Plant responses to salt stress}

Salinity is one of the major threats that drastically hinder plant growth and development. This leads to osmotic stress, oxidative stress and ionic stress resulting stunted growth accompanied by decreased leaf area and internode length, abscission and necrosis of leaves, and reduced yield. The extent of adverse effects varies widely among species. Plants adopt their defence strategies by osmotic adjustments, production of osmoprotectants (proline and soluble sugars), enzyme activation, $\mathrm{Na}+$ exclusion, ion $\left(\mathrm{Na}^{+} / \mathrm{K}^{+}\right)$homoeostasis and maintaining membrane potential to survive under salt stress.

\section{Signaling system under abiotic stresses}

The cellular signaling in plants under drought and salt stress follow one or the other following pathways-

\section{Osmotic/oxidative stress signaling}

Drought stress is caused due to high temperature. High temperature acts as a stressor that trigger various primary effects including plasmolysis at the cellular level, termed as strains. The signalling pathways for abiotic stresses are reported to cross-talk (converge) at multiple steps. In glycophytes, an over- production of compatible osmolytes e.g., proline, betaine, glycine, fructan, aminoalkanoic acid, alkaloids and polyols reduce the stress injury to plant cells. The transgenic plants with excess production of osmolytes showed improved salt and drought tolerance. The introduction of fructan synthesizing genes in tobacco plant increased the resistance to drought stress. These osmolytes might shield proteins from mis- 
folding and alleviate the harmful effects of Reactive Oxygen Species (ROS). $\gamma$-pyrroline5-carboxylate synthase ( $\gamma \mathrm{P} 5 \mathrm{CS})$ is the limiting enzyme for proline synthesis. Many genes that are induced or upregulated by osmotic stresses have been identified (Ingram and Bartel, 1996, Bray 1997, and Zhu et al., 1997). Up regulation of $\gamma$-pyrroline-5-carboxylate synthase $(\gamma \mathrm{P} 5 \mathrm{CS})$ has been reported to increase tolerance to salt or drought stresses. Over production of aminoalkanoic acid in plants might involve regulation by feedback inhibition. Tobacco plants expressing a mutated P5CS excelled proline biogenesis and showed increased salt tolerance.
Under oxidative stress, secondary stresses like ROS, e.g. hydroxyl radical and superoxide anions are produced higher level in plants and these are counteracted by compatible solutes to alleviate oxidative stress injury. The transgenic plants over-expressing ROS scavenging enzymes like peroxidase, suproxide dismutase, catalase and glutathione S-transferase (GST) showed increased tolerance to salt stress. In Arabidopsis, improved salt tolerance in pst mutants was achieved due to activation of ROS detoxification enzymes e.g., super oxide dismutase (SOD) and ascorbate peroxidase.

Fig.1 Schematic signaling pathways in plants under salt and drought stress

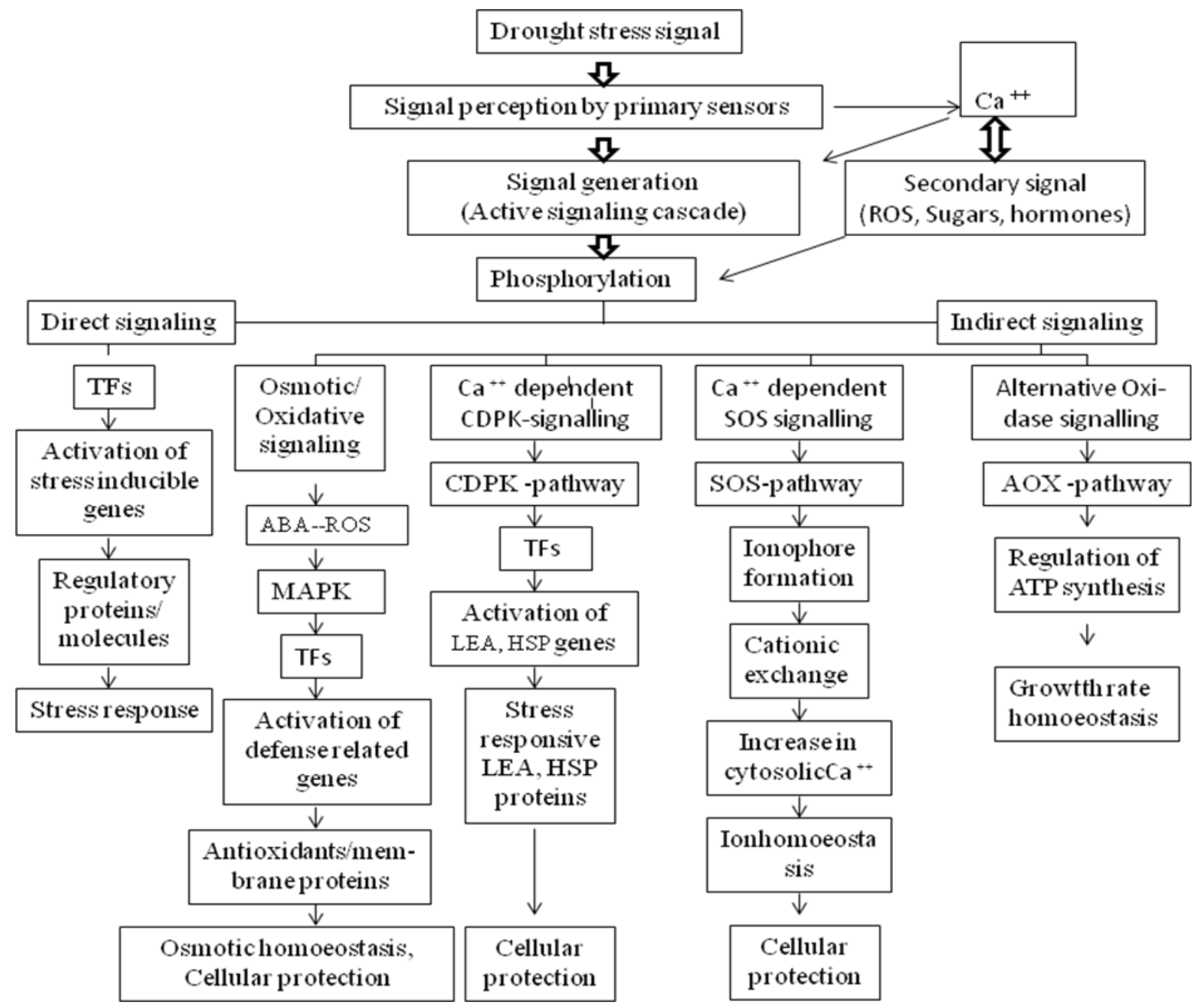


Under water deficit, ABA concentration in the cytosol is increased (Fig. 1) which acts as secondary signal molecule for activation of TFs and down stream effector genes (Zhu, 2002). The ABA stress inducible genes ( $\mathrm{rd} 22$, rd29A and $\mathrm{rd} 29 \mathrm{~B}$ ) respond to dehydration and these have ABA-responsive elements (ABRE) i.e. $(\mathrm{C} / \mathrm{T})$ ACGTGGC in their promoters. The rd29A promoter site contains both ABRE as well as DRE (drought responsive element: TACCGACAT) cis acting elements. TFs e.g., ABREBs (ABA responsive element binding proteins) and DREBs (drought responsive element binding proteins) regulate the rd29 gene through binding at ABRE and DRE sites of the promoter. Such regulatory architecture enable rd29 to dictate simultaneous tolerance to salt, cold and drought stress. Therefore, rd29 promoter sequence is in vogue used in genetic transformation intended for desiccation tolerance in crop plants.

Besides, plants speedily activate MAPK genes once exposed to multiple abiotic stress stimuli. The MAPK enzymes mediate signal transduction from the cell surface to the nucleus. These kinases appear to be widely used as osmolarity signaling modules and in several cases, these activate nuclear transcription factors to induce the expression of specific sets of genes. MAPK genes are extremely preserved in eukaryotic genomes and the resulting stress inducible (in response to drought and other environmental stresses) enzymes phosphorylate specific serine/ threonine residues on the relay proteins and regulate a range of cellular activities.

The MAPK phosphorylation cascade is a link between upstream receptors and downstream targets which regulate several vital cellular functions. The OsMSRMK2 transcripts (mRNA) are shown to accumulate within fifteen minutes of drought stress imposed. Thus, understanding of the MAPK cascade will give insight to understanding and finding the matter of drought/salt stress in agricultural crops.

\section{Ca2+dependent signaling that leads to activation of LEA-type genes}

A variety of calcium dependent protein kinases (CDPK) are associated with cell surface and these have role in signal perception and phosphorylation (Fig. 1). A calcium binding protein 'calmodulin' acts as sensors in the cytoplasm. In response to abiotic stresses, the cytosolic $\mathrm{Ca}^{+2}$ is increased which binds with calmodulin to serve as relay protein. Many other secondary messengers e.g., cyclic ADP ribose, nicotinic acid adenine dinucleotide phosphate (NADP) and inositol triphosphate (IP3) are also involved. In rice plants, a membrane associated CDPK is activated by cold treatment (Martin and Busconi, 2001). Overexpression of a CDPK gene 'osCDP7' results in increased cold and osmotic stress tolerance in rice. These calcium activated relay proteins in a series of steps carry the signal to the nucleus to activate Transcriptional Factors e.g. drought responsive elements (DREs) and cold responsive transcriptional factors (CRTs) to initiate transcription of mRNA from LEA or LEA like genes. These are highly expressed in seeds during desiccation stage and in vegetative tissues in response to water deficit (Baker et al., 1988). Overexpression of individual LEA genes has been reported to confer stress tolerance (drought and salinity) in transgenic rice (Xu et al., 1996).

\section{Ca2+dependent salt overlay sensitive (SOS) signaling}

This results ion homoeostasis by forming ionophores in the plasma membrane which allow free entry of $\mathrm{Ca}^{+2}$ into the cell in 
exchange of $\mathrm{Na}+$ ions that are excluded(Fi 1). Plants counteract salt stress by $\mathrm{Na}^{+}$extrusion, compartmentalization and controlled longdistance transport to aerial components, adequate $\mathrm{K}^{+}$nutrition and a favourable $\mathrm{K}^{+} / \mathrm{Na}^{+}$quantitative relation within the cytoplasm. Salt tolerant plants results immediate rise in cytosolic $\mathrm{Ca}^{2+}$ concentration to overcome salt injury. This was evidenced from expression and accumulation of vacuolar $\mathrm{Ca}^{2+}$ ATPase (encoding the Arabiodopsis ACA4 sequence) in yeast under salt stress. SOSs macromolecules are a distinct group protein kinase enzymes which are in fact, induced with increase in $\mathrm{Ca}^{+2}$ in the cytosol. Na extrusion is achieved by plasmamembrane localized $\mathrm{Na}^{+/} \mathrm{H}^{+}$antiporter SOS1. Mutations in SOS1 rendered the mutant plants sensitive to $\mathrm{Na}$. Arabidopsis plants are shown to increase $\mathrm{Na}^{+} / \mathrm{H}^{+}$antiporter activity in response to pretreatment with salt stress. Another mechanism to scale back accumulation of cytosolic $\mathrm{Na}^{+}$is achieved by the action of $\mathrm{Na}^{+} / \mathrm{H}^{+}$antiporters on the tonoplast. Transgenic Arabidopsis plants overexpressing the vacuolar $\mathrm{H}^{+}$ pyrophosphatase, AVP1 showed increased tolerance to salt stress. Similarly, overexpression of one of the vacuolar $\mathrm{Na}^{+} / \mathrm{H}^{+}$ antiporters, At NHX1, increased the salt tolerance of the Arabidopsis plants. During abiotic stresses, plants undergo transient increase in cytosolic $\mathrm{Ca}^{2+}$ coupled with downstream signaling events to mediate stress adaptation. In Arabidopsis salt stress signaling, the $\mathrm{Ca}^{2+}$ signal is perceived by the calcineurin- $\beta$-like $\mathrm{Ca}^{2+}$ sensor SOS3. While, the activated SOS2 might modulate the activity of particle transporters like SOS1 and HKT1.

\section{Alternative oxidase signaling (Cyanide resistant respiratory signaling)}

This signaling system induce alternative oxydase (AOX) - an additional enzyme involved in the respiration chain resistant to cyanide in higher plants (Fig. 1). AOX is reported to be expressed at wide range of developmental stages, and at biotic and abiotic stress conditions. AOX as a small family of nuclear genes (AOX1, AOX2a, AOX2b) (Considine et al., 2002). AOX1 is common in both mono and dicotyledons whereas, AOX2 genes are usually constitutively or developmentally expressed in dicotyledons only (Saisho et al., 2001a, 2001b). The stress signaling molecule $\mathrm{H}_{2} \mathrm{O}_{2}$ inducing the expression of AOX1 gene is perceived in the mitochondria and then transmitted to the nucleus (McIntosh et al., 1998) and led to upregulated expression of AOX1 under cold stress to enable the cell to cope up with altered metabolic conditions. Over expression of AOX1 decreased mitochondrial ROS formation (Maxwell et al., 1999). However, addition of antioxidants lowered the intracellular ROS level and inhibited AOX1 gene expression (Maxwell et al., 2002). AOX enable plant to maintain energy balance or stable energy under stressful environments and consequently able to maintain growth rate homoeostasis by flexible control on ATP synthesis (Moore et $a l .$, 2002). Robson and Vanlerberghe (2002) underline the physiological role of AOX as 'survival protein' to cope with adverse environments. Expression of AOX in response to cold stress has been demonstrated in green pepper (Purvis and Shewfelt, 1993).

Plants adopt variety of mechanisms to survive under drought and salt stress. Drought and salt tolerance as such are complex traits and many often a series of events (signalling pathways) underlying their mechanisms cross talk with each other and also other abiotic stresses e.g. cold and heat stresses. Plants respond and adapt their physiology to such stresses by number of mechanisms at physiological, biochemical and molecular level. As a result, a variety of genes are 
induced to combat the stresses directly or many often indirectly by regulating the gene expression (Shinozaki et al., 2003). A number of signalling elements have been identified by various researchers. However, their functional significance in each crop need to be explored and validated by fine tuning plant sensing and signaling systems under abiotic stresses.

\section{Acknowledgement}

The authors sincerely acknowledge and thank all researchers for their valuable contributions included in the text as references.

\section{References}

Baker. J., Steele, C., Dure, L. 1988. Sequence and characterization of 6 LEA proteins and their genes from cotton. Plant Mol. Biol., 11: 277-29.

Basu, N., Arshad, N. and Visweswariah, S.S. 2010. Receptor guanylyl cyclase C (GC-C): Regulation and signal transduction. Molecular and Cellular Biochem., 334: 67-80.

Bray, E. A. 1997. Plant responses to water deficit. Trends in plant science, 2: 4854.

Considine, M.J., Holtzapffel, R., Day, D.A., Whelan, J. and Millar, A.H. 2002. Molecular distinction between alternative oxidase from monocots and dicots. Plant physiol, 129: 949-953.

Eyster, K.M. 2007. New paradigms in signal transduction.

Biochemical Pharmacology, 73:1511-1519.

Ingram, J. and Bartel, D. 1996. The molecular basis of dehydration tolerance in plants. Annu. Rev. Plant Physiol. Plant Mol. Biol., 47, 377-403.

Martín, M.L. and Busconi, L. 2001. A rice membrane-bound calcium-dependent protein kinase is activated in response to low temperature. Plant Physiol., 125 (3): 1442-1449.
Maxwell, D.P., Nickels, R. and McIntosh, L. 2002. Evidence of mitochondrial involvement in the transduction of signals required for the induction of genes associated with pathogen attack and senescence. Plant J., 29:269-279.

Maxwell, D.P., Wang, Y. and McIntosh, L. 1999. The alternative oxidase lowers mitochondrial reactive oxygen production in plant cell. Proc. Natl. Aca. Sci., USA, 96:8271-8276.

McIntosh, I., Eichler, G., Gray, G., Maxwell, D.., Nickels, R. and Wang, Y. 1998. Biochemical and genetic controls exerted by plant mitochondria. Biochem. Biophys Acta, 1365: 278-284.

Menon, A.R. and Durakovic, C. 2014. Signal perception and transduction in plants. Periodicals of Engineering and Natural Sciences, 2(2): 15-29.

Moore, A.L., Albury, M.S., Crichton, P.G. and Affourtit, C. 2002. Function of the alternative oxidase: Is it still a scavenger? Trends Plant. Sci., 7:478481.

Purvis, A.C. and Shewfelt, R.J. 1993. Does the alternative pathway ameliorate chilling injury in sensitive plant tissues? Physiol. Plant, 88: 712-718.

Robson, C.A. and Vanlerberghe, G.C. 2002. Transgenic plant cells lacking mitochondrial alternative oxidase have increased susceptibility to mitochondria-dependent and independent pathways of programmed cell death. Plant Physiol., 129: 19081920.

Saisho, D., Nakazono, M., and Hirai, A. 2001a. ATP synthesis inhibitors as well as respiratory inhibitors increase steadystate level of alternative oxidase mRNA in Arabidopsis thaliana. J. Plant Physiol., 158: 241-245.

Saisho, D., Nakazono, M., Lee, K, Tsutsumi, N., Akita, S. and Hirai, A. 2001b. The gene for alternative oxidase -2(AOX-2) 
from Arabidopsis thaliana consists of five exons unlike other AOX genes and is transcribed at an early stage during germination. Genes Genet. Systems, 76: 89-97.

Xu, D., Duan, X., Wang, B., Hong, B, Ho, T.H.D., and Wu, R.. 1996. Expression of a late embryogenesis abundant protein gene, HVA1, from Barley confers tolerance to water deficit and salt stress in transgenic rice. Plant Physiol., 110(1): 249-257.
Yang, T. and Poovaiah, B.W. 2003. Calcium/calmodulin -mediated signal network in plants. Trends in Plant Science, 8: 505-512.

Zhu, J. K. 2002. Salt and drought stress signal transduction in plants. Annu. Rev. Plant Biol., 53, 247-273.

Zhu, J. K., Hasegawa, P. M. and Bressan, R. A.1997. Molecular aspects of osmotic stress in plants. CRC Crit. Rev. Plant Sci., 16, 263-277.

\section{How to cite this article:}

Swapan K. Tripathy, Gyanisha Nayak, Jagruti Naik, Monalisa Patnaik, Asit P. Dash, Dibya B. Sahoo and Prusti, A.M. 2019. Signal Transduction in Plants under Drought and Salt Stress-An Overview. Int.J.Curr.Microbiol.App.Sci. 8(08): 318-325.

doi: https://doi.org/10.20546/ijcmas.2019.808.036 